\title{
Utility Valuation of Multiname Credit Derivatives and Application to CDOs
}

\author{
Ronnie Sircar* Thaleia Zariphopoulou ${ }^{\dagger}$
}

First draft: July 2006; revised September 2007 \& July 2008

\begin{abstract}
We study the impact of risk-aversion on the valuation of credit derivatives. Using the technology of utility-indifference pricing in intensity-based models of default risk, we analyze resulting yield spreads in multiname credit derivatives, particularly CDOs. We study first the idealized problem with constant intensities where solutions are essentially explicit. We also give the large portfolio asymptotics for this problem. We then analyze the case where the firms have stochastic default intensities driven by a common factor, which can be viewed as another extreme from the independent case. This involves the numerical solution of a system of reaction-diffusion PDEs. We observe that the nonlinearity of the utility-indifference valuation mechanism enhances the effective correlation between the times of the credit events of the various firms leading to non-trivial senior tranche spreads, as often seen from market data.
\end{abstract}

Keywords: Credit derivatives, indifference pricing.

\section{Introduction}

Defaultable instruments, or credit-linked derivatives, are financial securities that pay their holders amounts that are contingent on the occurrence (or not) of a default event such as the bankruptcy of a firm or non-repayment of a loan. The market in credit-linked derivative products has grown astonishingly, from $\$ 631.5$ billion global volume in the first half of 2001 , to above $\$ 12$ trillion through the first half of $2005^{1}$. The growth from mid-2004 through mid-2005 alone was $128 \%$. They now account for approximately $10 \%$ of the total OTC derivatives market.

Despite the popularity and ever-increasing complexity of credit risk structured products, the quantitative technology for their valuation (and hedging) has lagged behind. This is largely due to the high-dimensionality of the basket derivatives, which are typically written on hundreds of underlying names; consequently, the computational efficiency of any valuation procedure severely limits model choice.

A major limitation of many approaches is the inability to capture and explain high premiums observed in credit derivatives markets for unlikely events, for example the spreads quoted for senior tranches of CDOs written on investment grade firms. The approach explored here is to explain such phenomena as a consequence of tranche holders' risk aversion, and to quantify this through the mechanism of utility-indifference valuation.

*ORFE Department, Princeton University, E-Quad, Princeton NJ 08544; sircar@princeton.edu. Work partially supported by NSF grant DMS-0456195.

${ }^{\dagger}$ Departments of Mathematics and Information, Risk and Operations Management, The University of Texas at Austin, Austin, TX 78712, zariphop@math.utexas.edu. Work partially supported by NSF grants DMS-0456118 and DMS-0091946.

${ }^{1}$ Source: ISDA data reported at http://www.credit-deriv.com/globalmarket.htm 


\section{Valuation Mechanisms}

In complete financial market environments, such as in the classical Black-Scholes model, the payoffs of derivative securities can be replicated by trading strategies in the underlying securities, and their prices are naturally deduced from the value of these associated portfolios. However, once non-traded risks such as unpredictable defaults are considered, perfect replication and, therefore, risk elimination breaks down, and alternative ways are needed for the quantification of risk and assignation of price.

One approach is to use market derivatives data, when available, to identify which of the many feasible arbitrage free pricing measures is consistent with market prices. In a different direction, valuation of claims involving nontradable risks can be based on optimality of decisions once this claim is incorporated in the investor's portfolio. Naturally, the risk attitude of the individual needs to be taken into account, and this is typically modeled by a concave and increasing utility function $U$. In a static framework, prices are determined through the certainty equivalent, otherwise known as the principle of equivalent utility $[5,16]$. The utility-based value of the claim, written on the risk $Y$ and yielding payoff $C(Y)$, is

$$
\nu(C)=U^{-1}\left(\mathbb{E}_{\mathbb{P}}\{U(C(Y))\}\right) .
$$

Note that the arbitrage free price and the certainty equivalent are very different. The first is linear and uses the risk neutral measure. The certainty equivalent price is nonlinear and uses the historical assessment of risks.

Prompted by the ever-increasing number of applications (event risk sensitive claims, insurance plans, mortgages, weather derivatives, etc.), considerable effort has been invested in analyzing the utility-based valuation mechanism. Due to the prevalence of instruments dependent on non-market risks (like default), there is a great need for building new dynamic pricing rules. These rules should identify and price unhedgeable risks and, at the same time, build optimal risk monitoring policies. In this direction, a dynamic utility-based valuation theory has been developed producing so-called indifference prices. The mechanism is to find the price at which the writer (buyer) of the claim is indifferent in terms of maximum expected utility between holding or not holding the derivative. Specification of the indifference price requires understanding how investors act optimally with or without the derivative. These issues are naturally addressed through stochastic optimization problems of utility maximization. We refer to $[22,23]$ and $[7]$ as classical references in this area. The indifference approach was initiated for European claims by Hodges and Neuberger [19] and further extended by Davis et al. [9].

\section{Credit Derivatives}

As well as single-name securities such as credit default swaps (CDSs), in which there is a relatively liquid market, basket, or multi-name products have generated considerable OTC activity. Typical of these are collateralized debt obligations (CDOs) whose payoffs depend on the default events of a basket portfolio of up to 300 firms over a number of years. As long as there are no defaults, investors in CDO tranches enjoy high yields, but as defaults start occurring they affect first the high-yield equity tranche, then the mezzanine tranches and perhaps on to the senior and supersenior tranches. See Davis and Lo [8] or Elizalde [13] for a concise introduction. Even more exotic contracts are $\mathrm{CDO}^{2}$ s, which depend on baskets of CDO tranches, though the market in these has thinned considerably since 2005 .

The focus of modeling in the credit derivatives industry has been on correlation between default times. Partly this is due to the adoption of the one-factor Gaussian copula model as industry 
standard and the practice (up till recently) of analyzing tranche prices through implied correlation. This revealed that traded prices of senior tranches could only be realised through these models with an implausibly high correlation parameter, the so-called correlation smile. After the simultaneous downgrades of Ford and General Motors in May 2005, the standard copula model sometimes could not even fit the data.

Rather than focusing on models with "enough correlation" to reproduce market observations via standard no-arbitrage pricing, the goal of this article is to understand the effects of risk aversion on valuation of basket credit derivatives. In particular, how does risk aversion value portfolios that are sensitive to the potential default of a number of firms, and so to correlation between these events? Does the nonlinearity of the indifference pricing mechanism enhance the impact of correlation? It seems natural that some of the prices or spreads seen in credit markets are due more to "crash-o-phobia" in a relatively illiquid market, with the effect enhanced nonlinearly in baskets. When senior tranches offer non-trivial spreads (albeit a few basis points) for protection against the default risk of $15-30 \%$ of investment grade US firms over the next five years, they are ascribing a seemingly large probability to "the end of the world as we know it". We seek to capture this directly as an effect of risk aversion leading to effective or perceived correlation, opposed to a mechanism of high direct correlation.

Taking the opposite angle, the method of indifference pricing should be attractive to participants in this still quite illiquid OTC market. It is a direct way for them to quantify the default risks they face in a portfolio of complex instruments, when calibration data is scarce. Unlike well-developed equity and fixed income derivatives markets where the case for traditional arbitrage-free valuation is more compelling, the potential for utility valuation to account for high-dimensionality in a way that is consistent with investors' fears of a cascade of defaults is a case for its application here.

For recent applications of indifference pricing to credit risk, see also Collin-Dufresne and Hugonnier [6], Bielecki et al. [3, 4], and Shouda [30]. Analysis of the single- and two-name cases appears in our previous work [31].

We begin in Section 2 with the full heterogeneous model with stochastic intensities. The combinatorial difficulties in this case when there are more than twenty or so firms mean that we need to specialize to a more manageable framework by assuming some homogeneity in the firms' dynamics. With this simplification, it become necessary only to keep track of the number of firms that have defaulted, but not which ones. We present examples from two extremes within this setup, first when intensities are identical and constant (Section 3), corresponding to independent default times; and second, when intensities are identical and stochastic (Section 4), so the default times are heavily correlated. Section 5 concludes.

\section{General Model}

A default event associated with a single firm occurs as in intensity-based models introduced by, among others, Artzner and Delbaen [1] , Madan and Unal [26], Lando [24] and Jarrow and Turnbull [21]. However our valuation mechanism incorporates information from the firm's stock price. Unlike in a traditional structural approach, default occurs at a non-predictable stopping time $\tau$ with stochastic intensity process $\lambda \geq 0$, which is correlated with the firm's stock price. These are sometimes called hybrid models (see, for example, [25]).

We suppose that there are $N$ firms, and let us fix firm $i$. We are given a probability space $(\Omega, \mathcal{F}, \mathbb{P})$, where $\mathbb{P}$ is the real-life (or statistical) probability measure, and a filtration $\left(\mathcal{G}_{t}^{i}\right)$. A convenient way to introduce the default times is to associate to each firm a non-explosive counting process $H_{t}^{(i)}$ that starts at zero. We assume $H^{(i)}$ has a historical intensity $\left(\lambda_{t}^{(i)}\right)$, a non-negative 
$\mathcal{G}_{t}^{i}$-predictable process with $\mathbb{E}\left\{\int_{0}^{t} \lambda_{s}^{(i)} d s\right\}<\infty$ for all $t>0$, such that $\left(H_{t}^{(i)}-\int_{0}^{t} \lambda_{s}^{(i)} d s\right)$ is a martingale. We are also given a sub-filtration $\mathcal{F}_{t} \subset \mathcal{G}_{t}^{i}$, and we will assume that $H^{(i)}$ is doubly stochastic driven by $\left(\mathcal{F}_{t}\right)$, meaning that $\lambda_{t}^{(i)}$ is $\mathcal{F}_{t}$-predictable, and, for $t>s$, conditional on $\mathcal{G}_{s}^{i} \vee \mathcal{F}_{t}$, the increment $H_{t}^{(i)}-H_{s}^{(i)}$ is Poisson distributed with parameter $\int_{s}^{t} \lambda_{u}^{(i)} d u$. The default time $\tau_{i}$ of firm $i$ is then defined by

$$
\tau_{i}=\inf \left\{t>0 \mid H_{t}^{(i)}>0\right\} .
$$

It is also useful to think of default as follows. Let $\left\{\xi_{i}\right\}_{i=1, \cdots, n}$ be independent standard exponential random variables on the same probability space, independent of the intensities $\lambda^{(i)}$. The default time $\tau_{i}$ of the $i$ th firm is defined by

$$
\tau_{i}=\inf \left\{t: \int_{0}^{t} \lambda_{s}^{(i)} d s=\xi_{i}\right\}
$$

the first time the cumulated intensity reaches the random draw $\xi_{i}$. Here we restrict to the special class of doubly stochastic default times that may be correlated only through their intensity processes. For more general constructions of default times, and technical details on intensities, we refer, for instance, to [4].

As is common in credit applications, we suppose the filtration $\left(\mathcal{F}_{t}\right)$ is generated by some factor process $Y$. The $i$ th firm has default time $\tau_{i}$, the first jump time of the Cox process $H^{(i)}$ with intensity $\lambda_{i}\left(Y_{t}\right)$, where $Y \in \mathbb{R}^{p}$ is a $p$-dimensional intensity driving factor, and the $\lambda_{i}$ are smooth non-negative bounded functions. We take $Y$ to be a diffusion driven by the $p$-dimensional Brownian motion $B$ :

$$
d Y_{t}=b\left(Y_{t}\right) d t+a\left(Y_{t}\right) d B_{t} .
$$

The firms' stock prices processes $\left(S^{(i)}\right)$ follow geometric Brownian motions:

$$
\frac{d S_{t}^{(i)}}{S_{t}^{(i)}}=\left(r+\mu_{i}\right) d t+\sigma_{i} d W_{t}^{(i)},
$$

where $\left(W^{(i)}\right)$ are (in general correlated) Brownian motions, $r \geq 0$ is the assumed constant interest rate, the $\mu_{i}$ are excess growth rates, and $\sigma_{i}>0$ are the volatilities. In applications, it is natural to expect that the model is specified in such a way that $\lambda_{i}\left(Y_{t}\right)$ and $S_{t}^{(i)}$ are negatively correlated: a firm's default risk tends to rise when its stock price falls.

\subsection{Maximal Expected Utility Problem}

Let $T<\infty$ denote our finite fixed horizon, chosen later to coincide with the expiration date of the derivatives contracts of interest. We begin with the Merton problem without the credit derivative. Each firm's stock is available for trading by the investor until it defaults, when the holding in that stock has to be liquidated and re-invested in the remaining stocks (if any) and the bank account. For simplicity, we do not explicitly model further loss to the stock holder upon default. While the exact timing of default comes as a surprise, in models where the intensity process is negatively correlated with the stock price process, the value of the investor's stock holding is likely to diminish leading up to default. More precise extended models could incorporate a jump downwards in the stock value on default.

The investor's control process is $\pi_{t}=\left(\pi_{t}^{(1)}, \cdots, \pi_{t}^{(N)}\right)$, where $\pi_{t}^{(i)}$ is the dollar amount held in the stock at time $t$, until $\tau_{i} \wedge T$. The control process $\pi$ is called admissible if it is nonanticipating (with respect to the full filtration $\mathcal{G}_{t}=\vee_{i=1}^{N} \mathcal{G}_{t}^{i}$ ), and satisfies the integrability constraint 
$E\left\{\int_{0}^{T}\left\|\pi_{s}\right\|^{2} d s\right\}<\infty$. The set of admissible policies is denoted by $\mathcal{A}$. In the case of heterogeneous dynamics and default intensities, it is necessary to keep track of which firms have defaulted and which are still alive. Let $I_{t} \subset\{1,2, \cdots, N\}$ denote the list of firms (ordered by number) which have not defaulted by time $t$.

Keeping the same notation $\pi$ for the discounted controls, the investor's discounted wealth process $X$ evolves according to

$$
d X_{t}=\sum_{i \in I_{t}} \pi_{t}^{(i)} \mu_{i} d t+\sum_{i \in I_{t}} \pi_{t}^{(i)} \sigma_{i} d W_{t}^{(i)}
$$

When there are $1 \leq n \leq N$ firms left, the index sets

$$
I_{n}^{k}=\left\{i_{1}(n, k), i_{2}(n, k), \cdots, i_{n}(n, k)\right\}, \quad k=1,2, \cdots,\left(\begin{array}{c}
N \\
n
\end{array}\right)
$$

describe all possible combinations of firms that have not yet defaulted. We shall work with exponential utility of discounted (to time zero) wealth. We are first interested in the optimal investment problem up to time $T$ of the investor who does not hold any derivative security. When there are no firms left, we have the Merton value function

$$
M^{(0)}(t, x)=-e^{-\gamma x} .
$$

For $n \geq 1$, we define the Merton value functions for the investor starting at time $t \leq T$ with initial wealth $x$, when the firms in $I_{n}^{k}$ are healthy and the others have defaulted. We have

$$
M^{\left(I_{n}^{k}\right)}(t, x, y)=\sup _{\left\{\pi^{(i)} \mid i \in I_{n}^{k}\right\}} \mathbb{E}\left\{-e^{-\gamma X_{T}} \mid X_{t}=x, Y_{t}=y, I_{t}=I_{n}^{k}\right\} .
$$

The Merton value functions $M^{\left(I_{n}^{k}\right)}(t, x, y)$ solve the following system of HJB PDEs

$$
\begin{aligned}
M_{t}^{\left(I_{n}^{k}\right)}+\sup _{\left\{\pi^{(i)} \mid i \in I_{n}^{k}\right\}} \mathcal{L}_{x y} M^{\left(I_{n}^{k}\right)}+\sum_{j \in I_{n}^{k}} \lambda_{j}(y)\left(M^{\left(I_{n}^{k} \backslash\{j\}\right)}-M^{\left(I_{n}^{k}\right)}\right) & =0 \\
M^{\left(I_{n}^{k}\right)}(T, x, y) & =-e^{-\gamma x},
\end{aligned}
$$

where $M^{\left(I_{n}^{k} \backslash\{j\}\right)}$ is the Merton value function when firm $j$ has dropped out, and $\mathcal{L}_{x y}$ denotes the infinitesimal generator of $(X, Y)$.

To lighten the notation, we will now assume $p=1$, so that the intensities are driven by a one-dimensional diffusion $Y$ whose Brownian driver, $B$ in (1), is also one-dimensional. We will denote by $\rho_{i j} \in(-1,1)$ the constant correlations between the Brownian motions driving the stock returns

$$
\mathbb{E}\left\{d W_{t}^{(i)} d W_{t}^{(j)}\right\}=\rho_{i j} d t, \quad i \neq j,
$$

and by $m_{i} \in(-1,1)$ the constant correlation between the Brownian motion $B$ driving the intensity and the driver of the $i$ th stock price:

$$
\mathbb{E}\left\{d W_{t}^{(i)} d B_{t}\right\}=m_{i} d t
$$

Definition 1 Let $\Sigma\left(I_{n}^{k}\right)$ be the $n \times n$ covariance matrix with entries

$$
\left(\Sigma\left(I_{n}^{k}\right)\right)_{j m}=\sigma_{i_{j}} \sigma_{i_{m}} \rho_{i_{j} i_{m}}, \quad \text { where } i_{j}=i_{j}(n, k), i_{m}=i_{m}(n, k),
$$

and let $\mu\left(I_{n}^{k}\right)$ be the $n \times 1$ vector of excess growth rates corresponding to the firms in the index set $I_{n}^{k}$. The diversity coefficient $D$ of the subset of firms $I_{n}^{k}$ is defined by

$$
D\left(I_{n}^{k}\right)=\mu\left(I_{n}^{k}\right)^{T} \Sigma\left(I_{n}^{k}\right)^{-1} \mu\left(I_{n}^{k}\right),
$$


which is a positive scalar under the assumptions on our model coefficients. We also introduce $c \in \mathbb{R}^{n}, E \in \mathbb{R}$ and $F \in \mathbb{R}_{+}$:

$$
c_{i}:=m_{i} \sigma_{i}, \quad E\left(I_{n}^{k}\right)=c\left(I_{n}^{k}\right)^{T} \Sigma\left(I_{n}^{k}\right)^{-1} \mu\left(I_{n}^{k}\right), \quad F\left(I_{n}^{k}\right)=c\left(I_{n}^{k}\right)^{T} \Sigma\left(I_{n}^{k}\right)^{-1} c\left(I_{n}^{k}\right) .
$$
PDEs

Re-writing (6), the Merton value functions $M^{\left(I_{n}^{k}\right)}(t, x, y)$ solve the following system of HJB

$$
\begin{aligned}
M_{t}^{\left(I_{n}^{k}\right)}+\mathcal{L}_{y} M^{\left(I_{n}^{k}\right)}+\sum_{j \in I_{n}^{k}} \lambda_{j}(y)\left(M^{\left(I_{n}^{k} \backslash\{j\}\right)}-M^{\left(I_{n}^{k}\right)}\right) & \\
-\frac{1}{2 M_{x x}^{\left(I_{n}^{k}\right)}}\left(D\left(I_{n}^{k}\right)\left(M_{x}^{\left(I_{n}^{k}\right)}\right)^{2}+2 E\left(I_{n}^{k}\right) a(y) M_{x y}^{\left(I_{n}^{k}\right)} M_{x}^{\left(I_{n}^{k}\right)}+a(y)^{2} F\left(I_{n}^{k}\right)\left(M_{x y}^{\left(I_{n}^{k}\right)}\right)^{2}\right) & =0, \\
M^{\left(I_{n}^{k}\right)}(T, x, y) & =-e^{-\gamma x},
\end{aligned}
$$

where $\mathcal{L}_{y}$ is the infinitesimal generator of $Y$ :

$$
\mathcal{L}_{y}=\frac{1}{2} a(y)^{2} \frac{\partial^{2}}{\partial y^{2}}+b(y) \frac{\partial}{\partial y}
$$

Here the value function $M^{\left(I_{n}^{k}\right)}$ is coupled through its PDE to the $n$ value functions corresponding to the subsets of size $n-1$ when one of the firms in $I_{n}^{k}$ has defaulted. The value function for $n=0$ is given by (4). The market information is contained in the coefficients $D\left(I_{n}^{k}\right), E\left(I_{n}^{k}\right)$ and $F\left(I_{n}^{k}\right)$.

We construct the solution of (8) in the standard way, by first making the transformation

$$
M^{\left(I_{n}^{k}\right)}(t, x, y)=-e^{-\gamma x} v^{\left(I_{n}^{k}\right)}(t, y) .
$$

Substituting into (8), we require the $v^{\left(I_{n}^{k}\right)}$ to satisfy the system of PDEs

$$
\begin{aligned}
v_{t}^{\left(I_{n}^{k}\right)}+\widetilde{\mathcal{L}}_{y} v^{\left(I_{n}^{k}\right)}-\alpha^{\left(I_{n}^{k}\right)}(y) v^{\left(I_{n}^{k}\right)}-\frac{1}{2} F\left(I_{n}^{k}\right) a(y)^{2} \frac{\left(v_{y}^{\left(I_{n}^{k}\right)}\right)^{2}}{v^{\left(I_{n}^{k}\right)}}+\sum_{j \in I_{n}^{k}} \lambda_{j}(y) v^{\left(I_{n}^{k} \backslash\{j\}\right)} & =0, \\
v^{\left(I_{n}^{k}\right)}(T, y) & =1,
\end{aligned}
$$

where

$$
\alpha^{\left(I_{n}^{k}\right)}(y)=\frac{1}{2} D\left(I_{n}^{k}\right)+\sum_{j \in I_{n}^{k}} \lambda_{j}(y)
$$

and

$$
\widetilde{\mathcal{L}}_{y}=\mathcal{L}_{y}-a(y) E\left(I_{n}^{k}\right) \frac{\partial}{\partial y} .
$$

The $n=0$ starting function is $v^{(0)}(t, y) \equiv 1$.

\subsection{Multi-Name Claims}

As an example of a basket claim, we consider a contract that pays at expiration time $T$ an amount depending on which firms have survived, that is, it has a European-style payoff $h\left(I_{T}\right)$, and does not depend on the timing of defaults. We tackle the more realistic case of CDO tranches in Section 3.

When there are no firms left, we have the claim holder's value function

$$
H^{(0)}(t, x, y)=-e^{-\gamma x+\operatorname{ch}(\emptyset)},
$$


where $\emptyset$ denotes the empty set, and $c=e^{-r T}$. For $n \geq 1$, we define the value functions for the investor starting at time $t \leq T$ with initial wealth $x$, when the firms in $I_{n}^{k}$ are healthy and the others have defaulted. We have

$$
H^{\left(I_{n}^{k}\right)}(t, x, y)=\sup _{\left\{\pi^{(i)} \mid i \in I_{n}^{k}\right\}}\left\{-e^{-\gamma X_{T}+\operatorname{ch}\left(I_{n}^{k}\right)} \mid X_{t}=x, Y_{t}=y, I_{t}=I_{n}^{k}\right\} .
$$

Proposition 1 The claim holder's value functions are given by

$$
H^{\left(I_{n}^{k}\right)}(t, x, y)=-e^{-\gamma x} w^{\left(I_{n}^{k}\right)}(t, y),
$$

where the $w^{\left(I_{n}^{k}\right)}$ solve the system of PDEs:

$$
\begin{aligned}
w_{t}^{\left(I_{n}^{k}\right)}+\widetilde{\mathcal{L}}_{y} w^{\left(I_{n}^{k}\right)}-\alpha^{\left(I_{n}^{k}\right)}(y) w^{\left(I_{n}^{k}\right)}-\frac{1}{2} F\left(I_{n}^{k}\right) a(y)^{2} \frac{\left(w_{y}^{\left(I_{n}^{k}\right)}\right)^{2}}{w^{\left(I_{n}^{k}\right)}}+\sum_{j \in I_{n}^{k}} \lambda_{j}(y) w^{\left(I_{n}^{k} \backslash\{j\}\right)} & =0 \\
w^{\left(I_{n}^{k}\right)}(T, y) & =e^{-\gamma \operatorname{ch}\left(I_{n}^{k}\right)} .
\end{aligned}
$$

Proof: The arguments are identical as with the Merton cascade problem, with the minor alteration in the terminal conditions. In particular, $H^{\left(I_{n}^{k}\right)}$ satisfy the same system of PDEs as (8), but with the terminal condition

$$
H^{\left(I_{n}^{k}\right)}(T, x, y)=-e^{-\gamma x+\operatorname{ch}\left(I_{n}^{k}\right)} .
$$

The buyer's indifference price $p_{0}(y)$ of the credit derivative at time zero when $Y_{0}=y$, and all $N$ firms are alive is defined by

$$
H^{\left(I^{(N)}\right)}\left(0, x-p_{0}, y\right)=M^{\left(I^{(N)}\right)}(0, x)
$$

and therefore

$$
p_{0}(y)=\frac{1}{\gamma} \log \left(\frac{v^{\left(I^{(N)}\right)}(0, y)}{w^{\left(I^{(N)}\right)}(0, y)}\right) .
$$

However, there is a huge computational burden in terms of the number of value functions that have to be stored because of the combinatorial complexity. For $N$ larger than about thirty, this is essentially insurmountable at this level of generality.

\subsection{Symmetric Model}

For computational tractability, we need to assume a large degree of symmetry (or exchangeability) between the firms. One way to achieve this is as follows.

Assumption 1 All the parameters are the same, namely $\mu_{i} \equiv \mu$ and $\sigma_{i} \equiv \sigma$, the Brownian motions have correlation structure $\rho_{i j} \equiv \rho$ and $m_{i} \equiv m$, with the restrictions

$$
\rho>-\frac{1}{(N-1)} \text { and } m^{2}<\rho+\frac{(1-\rho)}{N} .
$$

Furthermore, the intensity functions are identical: $\lambda_{i}(y) \equiv \lambda(y)$. 
This is similar to what is assumed in the "industry standard" one-factor Gaussian copula. The conditions (15) guarantee that the Brownian motions have a legitimate correlation structure (in other words that we have a positive definite covariance matrix), and are derived in Appendix A (see also[15]).

Under this assumption, we have that

$$
D\left(I_{n}^{k}\right)=D(n)=\frac{\mu^{2}}{\sigma^{2}} f(n), \quad E\left(I_{n}^{k}\right)=E(n)=\frac{m \mu}{\sigma} f(n), \quad F\left(I_{n}^{k}\right)=F(n)=m^{2} f(n),
$$

where we define

$$
f(n)=\frac{n}{\rho n+(1-\rho)}
$$

The details of the calculation are given in Appendix B. Observe that the assumed bound on $\rho$ in (15) implies that $\rho N+(1-\rho)>0$ and so for $1 \leq n \leq N$,

$$
f(n)=\frac{n}{\rho n+(1-\rho)} \geq \frac{n}{\rho N+(1-\rho)}>0 .
$$

We can think of $D$ as a diversity function that measures the increased opportunity as the size $n$ of the available market increases. As defined in (7), $D$ is like the square of the (multi-dimensional) Sharpe ratio. As expected it increases with $n$, but is concave as the marginal benefit of adding another firm diminishes with the number of firms. This is illustrated in Figure 1 for various $\rho$. For small $\rho$, the diversity coefficient grows rapidly with the number of firms. As the correlation increases, $D(n)$ levels off sooner, and in the extreme $\rho=1$, the curve is flat.

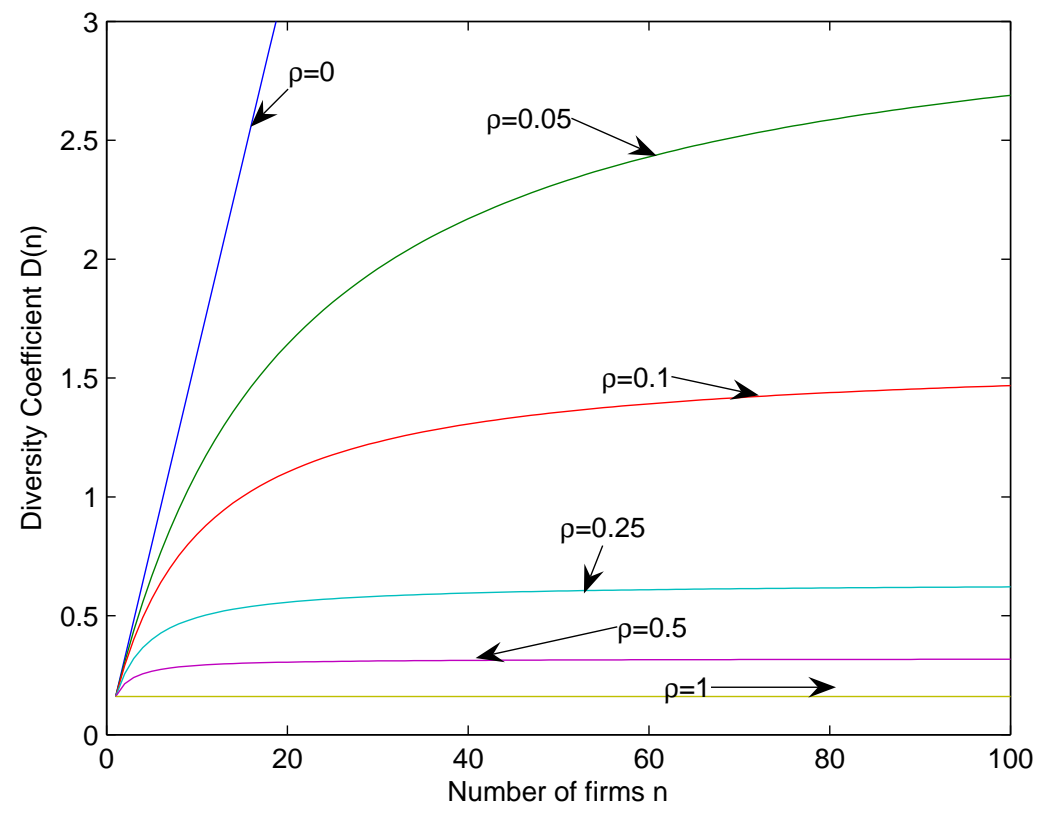

Figure 1: Diversity coefficient in (16) for various correlations $\rho$. The other parameters are $\mu=0.06$ and $\sigma=0.15$.

Under an additional assumption on the credit derivative, that its payoffs depend on the number of defaults but not their identity, we will need only to keep track of the number of firms alive at each time, not which particular ones. 


\section{Indifference Valuation of CDOs: Constant Intensities}

We next consider the indifference valuation of CDOs, first, to illustrate the approach, in the case when the intensities are constant. We return to stochastic intensities in Section 4. The underlying is a portfolio of $N$ defaultable bonds with total face value $Q$. The holder of a CDO tranche insures losses due to defaults between certain bounds (the attachment points characterizing the tranche). For some other approaches based on arbitrage pricing using intensity-based or copula models, we refer, for instance to $[8,10,11,17,18,20,28]$. A multi-dimensional structural model for loss distributions is studied in [14].

We are interested in the valuation of a CDO tranche with attachment points $K_{L}$ and $K_{U}$. These are percentages of the total portfolio value (notional) insured by the holder. For example $K_{L}=0 \%, K_{U}=3 \%$ corresponds to the equity tranche, and $K_{L}=3 \%, K_{U}=7 \%$ is typically the first mezzanine tranche. We assume the firms in the CDO have equal notionals, with $Q$ denoting the total notional. This assumption on the CDO structure is common in standardized contracts such as the CDX and iTraxx. For example, in the CDX CDOs, each of the 125 firms is protected up to losses of $\$ 80,000$, for a total notional of $Q=\$ 10$ million.

The tranche holder receives a yield $R \%$ (assumed paid continuously in our framework) on his part of the notional, which is initially $\left(K_{U}-K_{L}\right) Q$, but decreases as the losses arrive, until his tranche is blown. At each loss between the limits of his tranche's responsibility, he pays "notional minus recovery". We want to find the yield $R$ such that he is indifferent between holding the tranche or not.

\subsection{Merton Problem}

With $\lambda$ a constant, the $y$ variable no longer appears in our value functions. Let $M^{(n)}(t, x)$ denote the value function when there are $n \in\{0,1, \cdots, N\}$ firms alive. We have $M^{(0)}(t, x)=-e^{-\gamma x}$, and in general, for $1 \leq n \leq N, M^{(n)}(t, x)$ solves

$$
\begin{aligned}
M_{t}^{(n)}-\frac{1}{2} D(n) \frac{\left(M_{x}^{(n)}\right)^{2}}{M_{x x}^{(n)}}+n \lambda\left(M^{(n-1)}-M^{(n)}\right) & =0, \\
M^{(n)}(T, x) & =-e^{-\gamma x},
\end{aligned}
$$

the analog of (8) under the symmetry assumptions. In the next proposition, we construct an explicit solution for $M^{(n)}$.

Proposition 2 The Merton value functions are given by

$$
M^{(n)}(t, x)=-e^{-\gamma x} v_{n}(t),
$$

where

$$
v_{n}(t)=c_{0}^{(n)}+\sum_{j=1}^{n} c_{j}^{(n)} e^{-\alpha_{j}(T-t)}
$$

and

$$
\alpha_{n}:=\frac{1}{2} D(n)+n \lambda .
$$


The coefficients $c_{j}^{(n)}$ are found from the recursion relations

$$
\begin{aligned}
c_{0}^{(n)} & =\frac{n \lambda}{\alpha_{n}} c_{0}^{(n-1)}, \quad n=2, \cdots, N \\
c_{j}^{(n)} & =\frac{n \lambda}{\left(\alpha_{n}-\alpha_{j}\right)} c_{j}^{(n-1)}, \quad j=1, \cdots, n-1 \\
c_{n}^{(n)} & =1-\sum_{j=0}^{n-1} c_{j}^{(n)},
\end{aligned}
$$

with initial data $c_{0}^{(1)}=\left(\lambda / \alpha_{1}\right)$.

PROOF: Inserting the form (20) into (19) leads to the system of ODEs

$$
\begin{aligned}
v_{n}^{\prime}-\alpha_{n} v_{n}+n \lambda v_{n-1} & =0 \\
v_{n}(T) & =1
\end{aligned}
$$

Inserting the expression (21) leads to the recurrence relations (23).

Remark 1 (i) Note that the $v_{n}$ are independent of the risk-aversion coefficient $\gamma$.

(ii) The recursion relations can of course be solved explicitly, for example,

$$
c_{0}^{(n)}=\frac{\lambda^{n} n !}{\left(\alpha_{1} \alpha_{2} \cdots \alpha_{n}\right)}
$$

but it is computationally more stable to generate them recursively or via the matrix exponential.

\subsection{Tranche Holder's Problem}

We assume a fractional recovery $q$, meaning each default results in a loss to the portfolio of $(1-$ $q)(Q / N)$. The tranche holder (or protection seller) pays out this amount if the loss is within his tranche (up to the limit of the tranche). Our state variables are $t, x$ and $n$, the number of firms currently healthy. It is convenient, and more standard, to define the portfolio loss when there are $n$ firms remaining, namely

$$
\ell_{n}=(1-q) \frac{(N-n)}{N}
$$

Given the lower and upper tranche attachment points $K_{L}$ and $K_{U}$, we define

$$
G(\ell)=\left(K_{U}-\ell\right)^{+}-\left(K_{L}-\ell\right)^{+} .
$$

Then $G\left(\ell_{n}\right) \times Q$ is the remaining notional the tranche holder insures when there are $n$ firms still alive, and the capital on which he receives the tranche premium. The structure of the function $G$, being the difference of two put option payoffs, shows the tranche holder as having a "put spread" on the portfolio loss process with "strikes" $K_{L}$ and $K_{U}$.

Let $H^{(n)}(t, x)$ denote the tranche holders value function when $n$ firms are left. We assume he receives a cash flow at the rate $R e^{r t}$ at time $t$ on his remaining notional, where $R$ is the tranche premium to be found. The $e^{r t}$ factor is convenient to cancel the time variable out of the ODEs that follow. 
Then, in between default times, his discounted wealth process $X$ (depending on $n$ ) follows

$$
d X_{t}=\left(\sum_{i \in I_{t}} \pi_{t}^{(i)} \mu_{i}+R Q G\left(\ell_{n}\right)\right) d t+\sum_{i \in I_{t}} \pi_{t}^{(i)} \sigma_{i} d W_{t}^{(i)}
$$

To describe the protection payments made by the tranche holder when losses hit his tranche, we define

$$
g_{n}=G\left(\ell_{n}\right)-G\left(\ell_{n-1}\right) .
$$

Then $Q g_{n}$ is the payment made by the tranche holder if the number of firms remaining drops from $n$ to $n-1$ (that is, the loss increases from $\ell_{n}$ to $\ell_{n-1}$ ).

The HJB equation for $H^{(n)}(t, x)$ is

$$
\begin{aligned}
H_{t}^{(n)}-\frac{1}{2} D(n) \frac{\left(H_{x}^{(n)}\right)^{2}}{H_{x x}^{(n)}}+R Q G\left(\ell_{n}\right) H_{x}^{(n)}+n \lambda\left(H^{(n-1)}\left(t, x-Q g_{n}\right)-H^{(n)}\right) & =0 \\
H^{(n)}(T, x) & =-e^{-\gamma x} .
\end{aligned}
$$

In the next proposition, we construct an explicit solution for $H^{(n)}$.

Proposition 3 The tranche holder's value functions are given by

$$
H^{(n)}(t, x)=-e^{-\gamma x} w_{n}(t),
$$

where

$$
w_{n}(t)=d_{0}^{(n)}+\sum_{j=1}^{n} d_{j}^{(n)} e^{-\beta_{j}(T-t)}
$$

and

$$
\beta_{n}=\frac{1}{2} D(n)+n \lambda+\gamma R Q G\left(\ell_{n}\right) .
$$

The coefficients $d_{j}^{(n)}$ are found from the recursion relations

$$
\begin{aligned}
d_{0}^{(n)} & =\frac{q_{n}}{\beta_{n}} d_{0}^{(n-1)} \\
d_{j}^{(n)} & =\frac{q_{n}}{\left(\beta_{n}-\beta_{j}\right)} d_{j}^{(n-1)}, \quad j=1, \cdots, n-1 \\
d_{n}^{(n)} & =1-\sum_{j=0}^{n-1} d_{j}^{(n)},
\end{aligned}
$$

with initial data $d_{0}^{(1)}=\left(q_{1} / \beta_{1}\right)$, and where $q_{n}=n \lambda e^{\gamma Q g_{n}}$.

PROOF: Inserting the form (30) into (29) leads to the system of ODEs

$$
\begin{aligned}
w_{n}^{\prime}-\beta_{n} w_{n}+q_{n} w_{n-1} & =0 \\
w_{n}(T) & =1 .
\end{aligned}
$$

Inserting the expression (31) leads to the recurrence relations (33). 


\subsection{Indifference Tranche Spread}

Having solved for $v$ and $w$ in Propositions 2 and 3, the indifference tranche spread at time zero, when all $N$ firms in the CDO are alive, is found by solving for $R$ such that

$$
w_{N}(0)=v_{N}(0)
$$

Note that while the $v_{n}$ are independent of the risk-aversion coefficient $\gamma$, the $w_{n}$ from the tranche holder's problem depend on $\gamma$ through the combination $\gamma Q$. The indifference tranche spread is a mapping

$$
R=R\left(N ; \lambda, D(\cdot) ; \gamma Q ; K_{L}, K_{U}, T\right) .
$$

In Figure 2, we illustrate, in the case $N=25$, the types of spreads obtained for various tranches as the risk-aversion coefficient varies. We use throughout the diversity coefficient $D$ given in (16), and fix the attachment points as those corresponding to CDX CDOs:

\begin{tabular}{||c|c|c||}
\hline Tranche & $K_{L}$ & $K_{U}$ \\
\hline \hline Equity & $0 \%$ & $3 \%$ \\
Mezzanine 1 & $3 \%$ & $7 \%$ \\
Mezzanine 2 & $7 \%$ & $10 \%$ \\
Mezzanine 3 & $10 \%$ & $15 \%$ \\
Senior & $15 \%$ & $30 \%$ \\
\hline
\end{tabular}

Usually the premium for the equity tranche is quoted as an upfront fee that is paid in addition to 500 basis points, but for simplicity, we just treat the first tranche like the others. The same
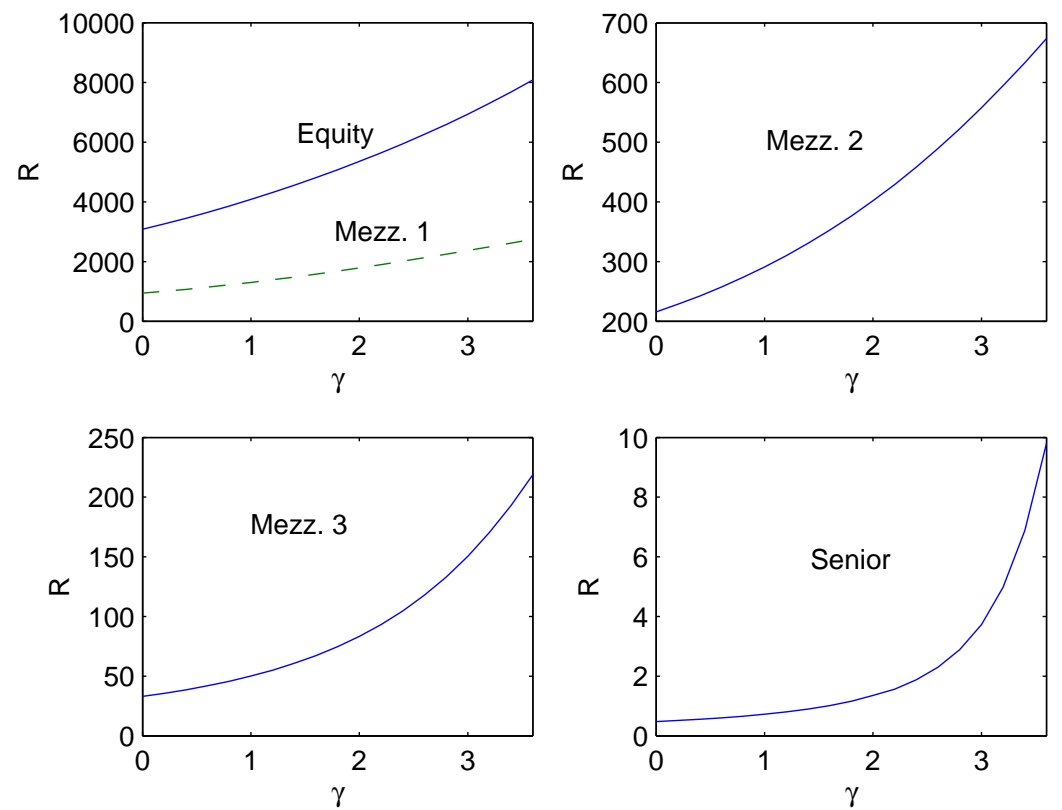

Figure 2: CDO indifference tranche spreads (in basis points) as functions of the risk-aversion coefficient $\gamma$. Number of firms $N=25$, and $\lambda=0.015$. The diversity coefficient $D$ is given by (16) with $\mu=0.07$, $\sigma=0.15$ and $\rho=0.3$. The recovery is $q=40 \%$, the interest rate $r=3 \%$ and $T=5$ years. The notional is normalized to 1 unit per firm, so $Q=N$.

qualitative features as in Figure 2 can be seen in Figure 3 for the case of $N=100$ firms, particularly 
the retarded sensitivity of the senior tranches to increases in risk-aversion. To remedy this problem, that is, to obtain more action in these tranches at more moderate risk-aversion levels, we return to stochastic intensities in Section 4.
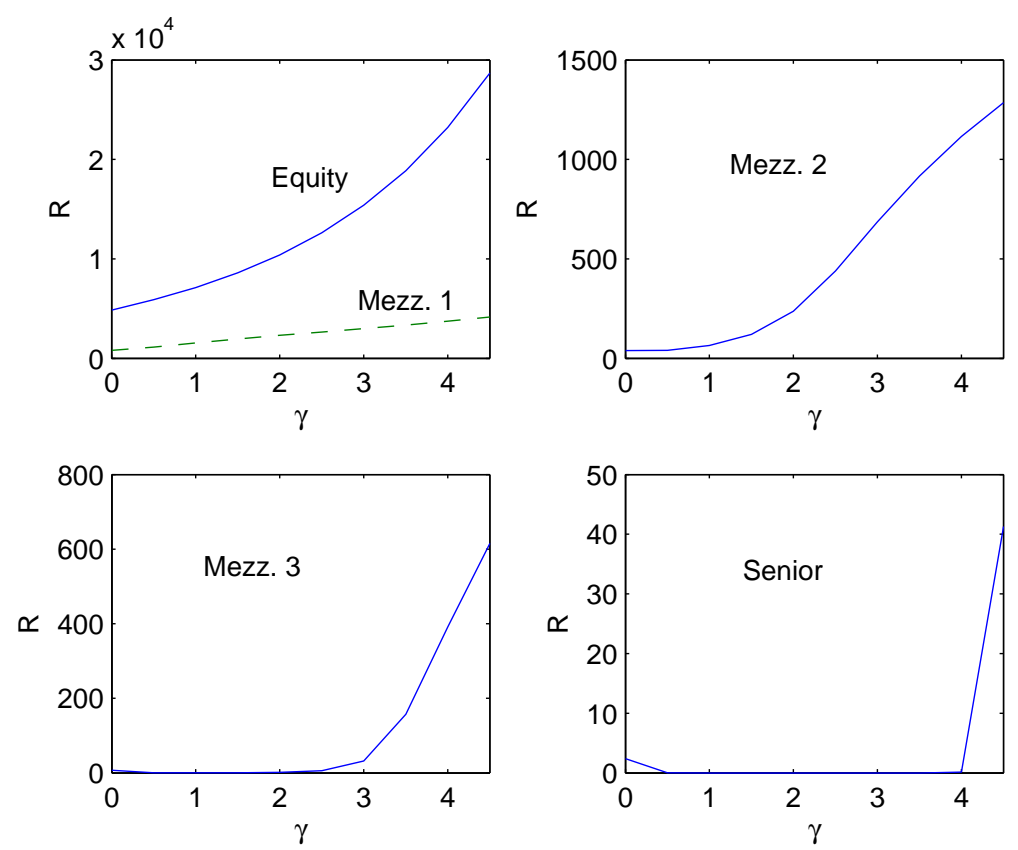

Figure 3: CDO indifference tranche spreads (in basis points) as functions of the risk-aversion coefficient $\gamma$. Number of firms $N=100$, all other parameters as in Figure 2.

As a preliminary and rough calibration exercise, we consider tranche spreads on the CDX from 10 March, 2008, well into the credit crisis that began in summer 2007. On this date, the (average of bid-ask) tranche premia are $\{67.38 \%, 727,403,204,164\}$, where the equity premium uses the "upfront plus 500 basis points" convention, and the others are quoted in basis points. We will ignore the equity tranche in this exercise, and the quarterly coupon payments, assuming continuous payment as in our analysis. Taking $N=125, \rho=0.3$ and the other parameters as in Figure 2, we find $\gamma$ for the four non-equity tranches that match the indifference value to the market spread. These are shown in the following table.

\begin{tabular}{||c|c|c|c||}
\hline Tranche & $K_{L}$ & $K_{U}$ & Implied $\gamma$ \\
\hline \hline Mezzanine 1 & $3 \%$ & $7 \%$ & $<10^{-4}$ \\
Mezzanine 2 & $7 \%$ & $10 \%$ & 2.38 \\
Mezzanine 3 & $10 \%$ & $15 \%$ & 3.59 \\
Senior & $15 \%$ & $30 \%$ & 4.77 \\
\hline
\end{tabular}

This fit shows that, within this crude model, the implied risk-aversion coefficient is increasing across tranches, relecting greater relative fear as regards the extreme losses the higher tranches represent. This is as we would expect (compare increased implied volatilities for out-of-the-money out options), and we leave further empirical understanding to a more detailed study.

\subsection{Large Portfolio Asymptotics}

It is naturally of interest to analyze the approximation that results from the limit as the number of firms $N$ goes to infinity. This relies of course on the homogeneity of the firms in the pool, as 
we have been assuming throughout this section. Large portfolio asymptotics have been computed at the level of the loss density in copula models, for example by Vasicek [33]; see also [29, Chapter 10]. For indifference valuation, we have to look directly at the two utility maximization problems, with and without the CDO.

The limit we consider is $N \rightarrow \infty$ with the total notional $Q$ fixed. Recall from Proposition 2 that the Merton value function $M^{(n)}(t, x)$ when there are $n$ firms undefaulted is given by $M^{(n)}(t, x)=$ $-e^{-\gamma x} v_{n}(t)$, where the $v_{n}$ satisfy the system of ODEs (25), which we re-write here as

$$
v_{n}^{\prime}-\frac{1}{2} D(n) v_{n}-n \lambda\left(v_{n}-v_{n-1}\right)=0, \quad v_{n}(T)=1 .
$$

Let $z=n / N \in[0,1]$ denote the fraction of firms still alive.

We shall take as given a smooth, increasing and concave diversity function $D(z)$, written as a function of the fraction $z$, that captures the benefits of having more firms to invest in. Although it is not the main goal of this paper, one could motivate the choice of a diversity function by considering an infinite-dimensional generalization of the model (2), namely

$$
\frac{d S_{t}^{(z)}}{S_{t}^{(z)}}=(r+\mu(z)) d t+\sigma(z) d W_{t}^{(z)},
$$

where $z \in[0,1]$ is a continuous parameter, and the $W^{(z)}$ are Brownian motions with some correlation structure. To generalize the symmetric model, we would take $\mu(z) \equiv \mu, \sigma(z) \equiv \sigma$ and

$$
\mathbb{E}\left\{d W_{t}^{(z)} d W_{t}^{\left(z^{\prime}\right)}\right\}=\rho d t, \quad z \neq z^{\prime} .
$$

When there is a fraction $z \leq 1$ of the firms left, the covariance function is given by

$$
\Sigma\left(z^{\prime}, z^{\prime \prime}\right)=\sigma^{2}\left[\rho+\delta\left(z^{\prime}-z^{\prime \prime}\right)\right], \quad z^{\prime}, z^{\prime \prime} \leq z,
$$

where $\delta$ is the Dirac delta function on $[0, z]$. Analogous to calculations for the discrete case in Appendix B, the inverse is given by

$$
\Sigma^{-1}\left(z^{\prime}, z^{\prime \prime}\right)=\frac{1}{\sigma^{2}}\left[\beta+\alpha \delta\left(z^{\prime}-z^{\prime \prime}\right)\right], \quad z^{\prime}, z^{\prime \prime} \leq z,
$$

where $\alpha$ and $\beta$ solve

$$
\begin{aligned}
\alpha+\rho \beta z & =1 \\
\beta+\rho \alpha+\rho \beta z & =0
\end{aligned}
$$

so that

$$
\int_{0}^{z} \Sigma(x, \xi) \Sigma^{-1}(\xi, y) d \xi=\delta(x-y)
$$

Then computing

$$
D(z)=\mu^{2} \int_{0}^{z} \int_{0}^{z} \Sigma^{-1}\left(z^{\prime}, z^{\prime \prime}\right) d z^{\prime} d z^{\prime \prime}=\frac{\mu^{2}}{\sigma^{2}}(\alpha+\beta z) z
$$

we obtain

$$
D(z)=\frac{\mu^{2}}{\sigma^{2}} \frac{z}{1+\rho(1-\rho) z} .
$$


Returning to the ODE system (35), we replace $v_{n}(t)$ by $V(t, z)$, where $z \in[0,1]$ is the continuous variable replacing $n / N$. Then applying

$$
n\left(v_{n}-v_{n-1}\right)=\frac{n}{N} \frac{\left(v_{n}-v_{n-1}\right)}{(1 / N)} \approx z V_{z},
$$

where subscript denotes partial derivative, we look for $V$ solving the first-order wave equation

$$
V_{t}-\frac{1}{2} D(z) V-\lambda z V_{z}=0,
$$

on $t<T$ and $0<z<1$, with $V(T, z)=1$. The solution is given by

$$
V(t, z)=\exp \left(-\frac{1}{2 \lambda}\left[I(z)-I\left(z e^{-\lambda(T-t)}\right)\right]\right)
$$

where

$$
I(z)=\int^{z} \frac{D(\xi)}{\xi} d \xi
$$

For the tranche holder's investment problem, recall from Proposition 3 that his value functions are given by $H^{(n)}(t, x)=-e^{-\gamma x} w_{n}(t)$, where the $w_{n}$ satisfy the system of ODEs (34), which we re-write here as

$$
w_{n}^{\prime}-\left(\frac{1}{2} D(n)+\gamma R Q G\left(\ell_{n}\right)\right) w_{n}-n \lambda\left(w_{n}-e^{\gamma Q g_{n}} w_{n-1}\right)=0 .
$$

We replace $w_{n}(t)$ by $W(t, z)$ solving the wave equation

$$
W_{t}-\left(\frac{1}{2} D(z)+\gamma Q\left(R \tilde{G}(z)-\lambda z \tilde{G}^{\prime}(z)\right)\right) W-\lambda z W_{z}=0,
$$

on $t<T$ and $0<z<1$, with $W(T, z)=1$, and where $\tilde{G}(z):=G((1-q)(1-z))$. The solution is given by

$$
W(t, z)=\exp \left(-\frac{1}{2 \lambda}\left[J(z)-J\left(z e^{-\lambda(T-t)}\right)\right]\right)
$$

where

$$
\begin{aligned}
J(z) & =-\frac{1}{2} I(z)+Q \gamma(\lambda \tilde{G}(z)-R \tilde{I}(z)) \\
\tilde{I}(z) & =\int^{z} \frac{\tilde{G}(\xi)}{\xi} d \xi .
\end{aligned}
$$

Finally, solving for the tranche premium $R$ such that

$$
W(0,1 ; R)=V(0,1)
$$

leads to

$$
R=\lambda \frac{\tilde{G}\left(e^{-\lambda T}\right)-\tilde{G}(1)}{\tilde{I}\left(e^{-\lambda T}\right)-\tilde{I}(1)} .
$$

From this formula, we see that the essentials of the indifference valuation, namely the risk aversion $\gamma$ and the diversity function $D$, have vanished, and so the large portfolio limit is rather crude. We end up with the approximation in which the pre-recovery loss process (the fraction of the portfolio lost up till time $t$ ) is trivially $L_{t}=1-e^{-\lambda t}$. Nonetheless, the large portfolio approximations (37) and (39) for the value functions with and without the tranche may be useful for other purposes, for example optimal investment in the presence of default risk, or hedging, but we do not explicitly consider those problems here. 


\section{Indifference Valuation of CDOs: Stochastic Intensities}

We return to the setting of Section 2, where intensities are stochastic, but with the symmetry assumptions of Section 2.3. To summarize, the firms have the same growth rates $\mu$, volatilities $\sigma$ and instantaneous correlations between returns $\rho$. Their default intensities are identically $\lambda\left(Y_{t}\right)$, driven by the one-dimensional diffusion $Y$ in (1), whose Brownian motion $B$ has the same correlation $m$ with any of the stock price Brownian motions $W^{(i)}$. As a consequence, the Merton value functions defined in (5) depend only on the number of firms alive and not which ones, and we write them $M^{(n)}(t, x, y)$. With the usual separation of variables $M^{(n)}(t, x, y)=-e^{-\gamma x} v^{(n)}(t, y)$, we have the analog of (10), namely

$$
\begin{aligned}
v_{t}^{(n)}+\widetilde{\mathcal{L}}_{y}^{(n)} v^{(n)}-\alpha_{n}(y) v^{(n)}-\frac{1}{2} f(n) a(y)^{2} m^{2} \frac{\left(v_{y}^{(n)}\right)^{2}}{v^{(n)}}+n \lambda(y) v^{(n-1)} & =0 \\
v^{(n)}(T, y) & =1
\end{aligned}
$$

where

$$
\alpha_{n}(y)=\frac{1}{2} D(n)+n \lambda(y)
$$

and

$$
\widetilde{\mathcal{L}}_{y}^{(n)}=\mathcal{L}_{y}-a(y) E(n) \frac{\partial}{\partial y}
$$

The $n=0$ starting function is $v^{(0)}(t, y) \equiv 1$. Observe that in the case of constant intensities where $y$ disappears as a variable, the PDE system (40) reduces to the ODE system (25) in Section 3.

Recall that for the tranche holder receiving a premium at rate $R e^{r t}$ at time $t$ on his remaining notional, the wealth dynamics are given by (28). When there are $n$ firms alive, the value function, denoted by $H^{(n)}(t, x, y)$ satisfies

$$
\begin{aligned}
H_{t}^{(n)}+\mathcal{L}_{y} H^{(n)}+n \lambda(y)\left(H^{(n-1)}\left(t, x-Q g_{n}, y\right)-H^{(n)}\right)+R Q G\left(\ell_{n}\right) H_{x}^{(n)} & \\
-\frac{1}{2 H_{x x}^{(n)}}\left(D(n)\left(H_{x}^{(n)}\right)^{2}+2 E(n) a(y) H_{x y}^{(n)} H_{x}^{(n)}+a(y)^{2} F(n)\left(H_{x y}^{(n)}\right)^{2}\right) & =0, \\
H^{(n)}(T, x, y) & =-e^{-\gamma x .}
\end{aligned}
$$

Making the transformation

$$
H^{(n)}(t, x, y)=-e^{-\gamma x} w^{(n)}(t, y)
$$

leads to the system

$$
\begin{aligned}
w_{t}^{(n)}+\widetilde{\mathcal{L}}_{y}^{(n)} w^{(n)}-\beta_{n}(y) w^{(n)}-\frac{1}{2} f(n) a(y)^{2} m^{2} \frac{\left(w_{y}^{(n)}\right)^{2}}{w^{(n)}}+n \lambda(y) e^{\gamma Q g_{n}} w^{(n-1)} & =0 \\
w^{(n)}(T, y) & =1,
\end{aligned}
$$

where $\beta_{n}(y)=\alpha_{n}(y)+\gamma R Q G\left(\ell_{n}\right)$. Observe that in the case of constant intensities where $y$ disappears as a variable, the PDE system (42) reduces to the ODE system (34) in Section 3.

\subsection{Reaction-Diffusion Systems}

The system (40) can be recast into a system of reaction-diffusion equations by the power transformation

$$
v^{(n)}(t, y)=\left(u^{(n)}(t, y)\right)^{\delta_{n}}, \quad \delta_{n}=\frac{1}{1-m^{2} f(n)} .
$$


Note that under the assumed bound on $m^{2}$ in (15),

$$
1-m^{2} f(n)>1-\left(\rho+\frac{(1-\rho)}{N}\right) f(n) \geq 1-\left(\rho+\frac{(1-\rho)}{n}\right) f(n)=0,
$$

and since $1-m^{2} f(n)<1$, we conclude that $\delta_{n}>1$.

Then, it is easy to check that for $n \geq 1, u^{(n)}(t, y)$ satisfies

$$
\begin{aligned}
u_{t}^{(n)}+\widetilde{\mathcal{L}}_{y}^{(n)} u^{(n)}-\frac{\alpha_{n}(y)}{\delta_{n}} u^{(n)}+\frac{n \lambda(y)}{\delta_{n}}\left(u^{(n-1)}\right)^{\delta_{n-1}}\left(u^{(n)}\right)^{1-\delta_{n}} & =0 \\
u^{(n)}(T, y) & =1,
\end{aligned}
$$

and $u^{(0)}(t, y) \equiv 1$.

Making the same type of transformation in (42)

$$
w^{(n)}(t, y)=\left(h^{(n)}(t, y)\right)^{\delta_{n}}
$$

leads to the reaction-diffusion system:

$$
\begin{aligned}
h_{t}^{(n)}+\widetilde{\mathcal{L}}_{y}^{(n)} h^{(n)}-\frac{\beta_{n}(y)}{\delta_{n}} h^{(n)}+\frac{n \lambda(y)}{\delta_{n}} e^{\gamma Q g_{n}}\left(h^{(n-1)}\right)^{\delta_{n-1}}\left(h^{(n)}\right)^{1-\delta_{n}} & =0 \\
h^{(n)}(T, y) & =1,
\end{aligned}
$$

and $h^{(0}(t, y) \equiv 1$. The trade-off between the incoming tranche premium and the outgoing protection payments is in the $\beta_{n}$ and $e^{\gamma Q g_{n}}$ coefficients respectively.

\subsubsection{Single-Name Case}

We consider first the case $n=1$, corresponding to only one firm left. Writing for the moment $u=u^{(1)}$, we have $u:[0, T] \times \mathbb{R} \rightarrow \mathbb{R}^{+}$solving the scalar reaction-diffusion equation

$$
\begin{aligned}
u_{t}+\widetilde{\mathcal{L}}_{y} u-\left(1-m^{2}\right)\left(\frac{\mu^{2}}{2 \sigma^{2}}+\lambda(y)\right) u+\left(1-m^{2}\right) \lambda(y) u^{-\left(\delta_{1}-1\right)} & =0 \\
u(T, y) & =1,
\end{aligned}
$$

where

$$
\delta_{1}-1=\frac{m^{2}}{1-m^{2}}, \quad \text { and } \quad \widetilde{\mathcal{L}}_{y}=\widetilde{\mathcal{L}}_{y}^{(1)}
$$

Similar equations arise in other utility problems in incomplete markets, for example, in portfolio choice with recursive utility [32], valuation of mortgage-backed securities [34] and life-insurance problems [2]. first with It is worth noting, however, that the reaction-diffusion equation (45) does not belong to the class of such equations with Lipschitz reaction term. Therefore, more detailed analysis is needed for establishing existence, uniqueness and regularity results. In the context of a portfolio choice problem with stochastic differential utilities, the analysis can be found in [32]. The equation at hand is slightly more complicated than the one analyzed there, in that the reaction term has the multiplicative intensity factor. Because $\lambda(\cdot)$ is taken to be bounded and Lipschitz, an adaptation of the arguments in [32] can be used to show that the reaction-diffusion problem (45) has a unique bounded and smooth solution. Furthermore, we have the following bounds.

Proposition 4 The solution $u(t, y)$ of the PDE problem (45) satisfies the bounds:

$$
e^{-\left(1-m^{2}\right) \frac{\mu^{2}}{2 \sigma^{2}}(T-t)} \leq u(t, y) \leq 1 \text {. }
$$


Proof: The test solution $\tilde{u} \equiv 1$ satisfies the terminal condition in (45). Testing this solution in the PDE for $u$, we have

$$
\tilde{u}_{t}+\widetilde{\mathcal{L}}_{y} \tilde{u}-\left(1-m^{2}\right)\left(\frac{\mu^{2}}{2 \sigma^{2}}+\lambda(y)\right) \tilde{u}+\left(1-m^{2}\right) \lambda(y) \tilde{u}^{-\left(\delta_{1}-1\right)}=-\left(1-m^{2}\right) \frac{\mu^{2}}{2 \sigma^{2}} \leq 0,
$$

so $\tilde{u}$ is a supersolution of (45), which implies $u \leq 1$. Next, testing $\tilde{u}=e^{-\left(1-m^{2}\right) \frac{\mu^{2}}{2 \sigma^{2}}(T-t)}$ gives

$$
\begin{aligned}
\tilde{u}_{t}+\widetilde{\mathcal{L}}_{y} \tilde{u}-\left(1-m^{2}\right)\left(\frac{\mu^{2}}{2 \sigma^{2}}+\lambda(y)\right) \tilde{u}+\left(1-m^{2}\right) \lambda(y) \tilde{u}^{-\left(\delta_{1}-1\right)} & =\left(1-m^{2}\right) \lambda(y)\left(\tilde{u}^{-\left(\delta_{1}-1\right)}-\tilde{u}\right) \\
& \geq 0,
\end{aligned}
$$

where the inequality follows from $\tilde{u} \leq 1$ and $\left(\delta_{1}-1\right)>0$. Hence $\tilde{u}$ is a subsolution of (45), which implies the lower bound in (46).

\subsubsection{Bounds in the Multiname Case}

Returning to the reaction-diffusion system (43) for $\left\{u^{(n)}(t, y), n=1,2, \cdots, N\right\}$, we establish the following bounds.

Proposition 5 The solution $u^{(n)}(t, y)$ of the PDE system (43) satisfies the bounds:

$$
e^{-\frac{1}{2} \frac{D(n)}{\delta_{n}}(T-t)} \leq u^{(n)}(t, y) \leq 1
$$

Proof: The case $n=1$ has already been established in Proposition 4. Suppose the bounds (47) hold for $n-1$. Then testing $\tilde{u}^{(n)} \equiv 1$ in (43), we find

$$
\begin{aligned}
\tilde{u}_{t}^{(n)}+\widetilde{\mathcal{L}}_{y}^{(n)} \tilde{u}^{(n)}-\frac{\alpha_{n}(y)}{\delta_{n}} \tilde{u}^{(n)}+\frac{n \lambda(y)}{\delta_{n}} & \left(u^{(n-1)}\right)^{\delta_{n-1}}\left(\tilde{u}^{(n)}\right)^{1-\delta_{n}} \\
& =-\frac{1}{2} \frac{D(n)}{\delta_{n}}+\frac{n \lambda(y)}{\delta_{n}}\left(\left(u^{(n-1)}\right)^{\delta_{n-1}}-1\right) \\
& \leq 0,
\end{aligned}
$$

using the upper bound $u^{(n-1)} \leq 1$ and $\delta_{n-1}>1$. Hence $\tilde{u}^{(n)} \equiv 1$ is a supersolution and we have the upper bound in (47). Similarly, testing $\tilde{u}^{(n)}=e^{-\frac{1}{2} \frac{D(n)}{\delta_{n}}(T-t)}$ in the PDE (43), we obtain

$$
\begin{aligned}
\tilde{u}_{t}^{(n)}+\widetilde{\mathcal{L}}_{y}^{(n)} \tilde{u}^{(n)}-\frac{\alpha_{n}(y)}{\delta_{n}} \tilde{u}^{(n)}+\frac{n \lambda(y)}{\delta_{n}} & \left(u^{(n-1)}\right)^{\delta_{n-1}}\left(\tilde{u}^{(n)}\right)^{1-\delta_{n}} \\
& =\frac{n \lambda(y)}{\delta_{n}} e^{-\frac{1}{2} \frac{D(n)}{\delta_{n}}(T-t)}\left(e^{-\frac{1}{2}(D(n-1)-D(n))(T-t)}-1\right) \\
& \geq 0
\end{aligned}
$$

using the lower bound on $u^{(n-1)}$. The last inequality follows because $D(n)$ is increasing. Thus we have a subsolution, which establishes the lower bound in (47).

These bounds could be used as a check on numerical or asymptotic approximations for the value functions $u^{(n)}$. We remark that establishing similar bounds for the tranche holder's value functions, $h^{(n)}$ in (44), is not so simple because of the factor $e^{\gamma Q g_{n}} \geq 1$ in front of the nonlinear term, which complicates the use of comparison principles. 


\subsection{Indifference Tranche Spreads}

To illustrate the effect of stochastic intensities (and, therefore, correlated default times) and utility valuation, we work with the model

$$
\begin{aligned}
d Y_{t} & =a\left(y_{\infty}-Y_{t}\right) d t+b d B_{t} \\
\lambda(y) & =\frac{k}{\pi}\left(\tan ^{-1}(y)+\frac{\pi}{2}\right) .
\end{aligned}
$$

So, $Y$ is a mean-reverting OU process and $\lambda$ a positive function bounded above by $k$. The choice of the model is necessarily ad hoc, but it is an example from a reasonable class of stochastic intensities that allows us to present the effects of indifference valuation in a computational case study.

We then solve the reaction-diffusion systems (43) and (44) numerically. The tranche premium is determined by finding $R$ such that

$$
u^{(N)}\left(0, Y_{0}\right)=h^{(N)}\left(0, Y_{0} ; R\right)
$$

For the numerical solutions, we use an implicit-explicit finite-difference scheme: implicit discretization of the linear part of the PDEs, and explicit on the nonlinear reaction term. For $N=25$ firms, the results are shown in Figure 4.
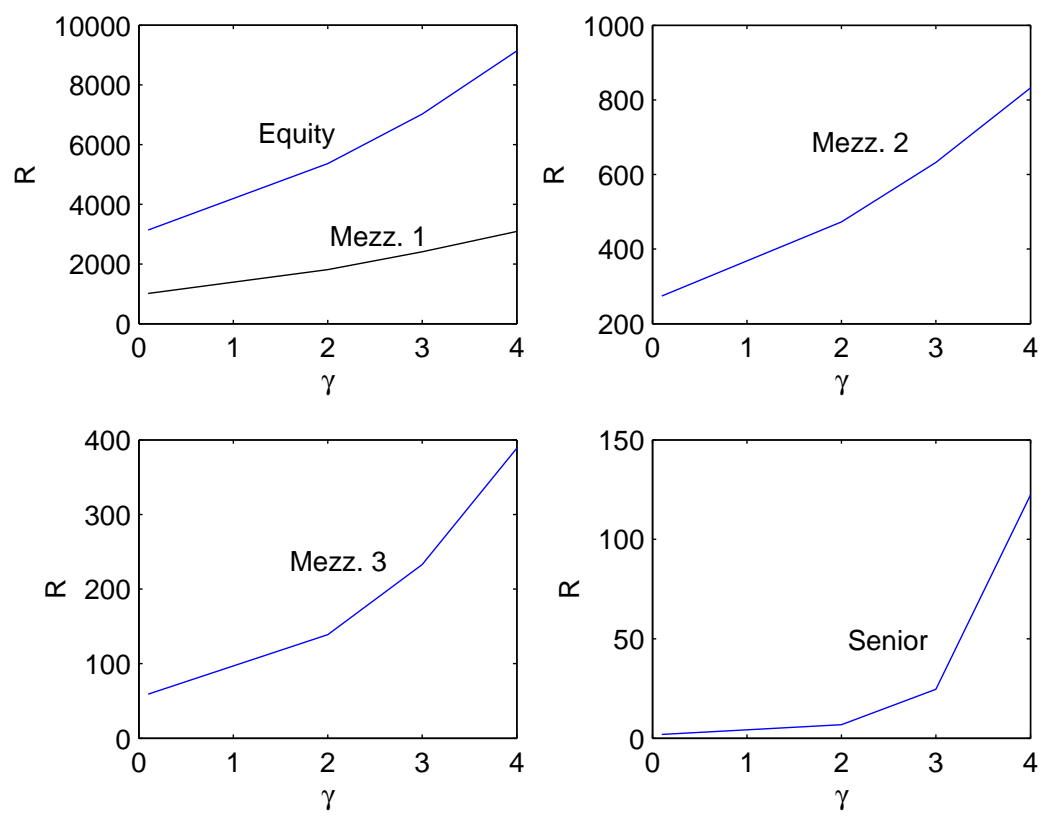

Figure 4: CDO indifference tranche spreads (in basis points) as functions of the risk-aversion coefficient $\gamma$. Number of firms $N=25$, and identical stochastic intensities $\lambda\left(Y_{t}\right)$ in equation (48) with $k=0.06$. The model coefficients are $\mu=0.07, \sigma=0.15$ and $\rho=0.3$. The recovery is $q=40 \%$, the interest rate $r=3 \%$ and $T=5$ years. The notional is normalized to 1 unit per firm, so $Q=N$.

Intensity-based arbitrage pricing is studied in [11, 27, 28], where it is demonstrated in a variety of models that it is difficult to fit the market-quoted senior tranche spread without overstating enormously the mezzanine tranches. As Figure 4 shows, these are better captured with utility valuation that reflects the specialization and relative illiquidity of the market in these derivatives. However stochastic intensity is important: in Figure 2 with constant intensities, we find essentially 
zero $(R<1)$ premium for the senior tranche with a risk aversion $\gamma<2$, while more pronounced premia of a few basis points $(R \approx 5$ for $\gamma=2$ and $R \approx 15$ for $\gamma=2.5$ ), as has been seen in some market data, can be accounted for with more moderate risk aversion coefficients as long as intensities are stochastic (Figure 4). This highlights the importance of using utility valuation to enhance already correlated default events.

\section{Conclusion}

The preceding analysis demonstrates that utility valuation produces non-trivial CDO tranche spreads and implied correlations within even the simplest of intensity-based models of default. It also incorporates equity market information (growth rates, volatilities of the non-defaulted firms) as well as investor risk aversion to provide a relative value mechanism for multi-name credit derivatives. To obtain non-trivial senior tranche spreads though, of the order seen in recent market quotes, stochastic intensities are needed.

It is of interest to generalize the basic model in a number of ways for greater descriptive precision. Recent research, for example [12], points out the importance of a varying interest rate and its impact on credit risk. Here we have not considered stochastic interest rates since this would increase the dimensionality of the problem and we would lose quantitative and qualitative tractability. Nonetheless a full computational implementation could be developed to incorporate a low-dimensional Markovian interest rate model.

Many issues remain for investigation: the effects of the various input parameters; the choice and estimation of different diversity coefficients that contain the impact of defaults on the diminishing investment opportunity set; the effect of time-varying or stochastic risk-aversion; efficient computation and analysis of the fully heterogeneous case; and large portfolio asymptotics of indifference values with stochastic intensities. A related problem is optimal static-dynamic hedging of CDO tranches, combining dynamic trading strategies in the underlying firms' stocks, and static positions in CDSs.

\section{A Restrictions on Common Correlation Coefficients}

In the symmetric model described in Section 2.3, the common correlation coefficients $\rho$ and $m$ have to be chosen such that the ensuing $(N+1) \times(N+1)$ covariance matrix (omitting the $\sigma^{2}$ factor)

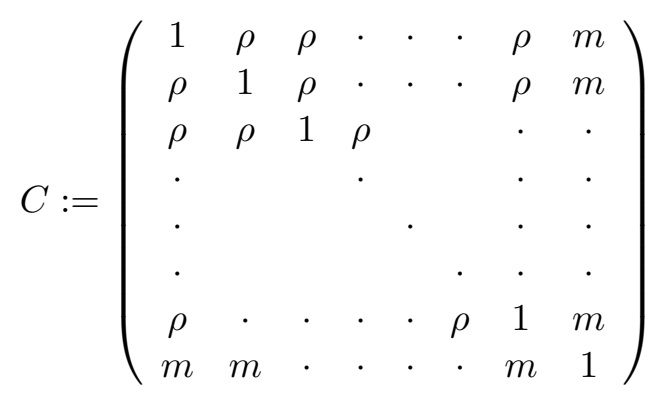

is indeed positive definite. First consider the upper $N \times N$ block, call it $B$, which must also be positive definite. This is a matrix with ones on the diagonal and $\rho$ everywhere else and can be written

$$
B=(1-\rho) \mathcal{I}_{N}+\rho \mathcal{J}_{N},
$$


where $\mathcal{I}_{N}$ is the $N \times N$ identity matrix, and $\mathcal{J}_{N}$ is the $N \times N$ matrix of all ones. Let $x \in \mathbb{R}^{N}$ be an arbitrary vector. Then we have

$$
x^{T} B x=(1-\rho)\|x\|_{2}^{2}+\rho\left(\mathbf{1}^{T} x\right)^{2},
$$

where $\mathbf{1} \in \mathbb{R}^{N}$ is the column vector of ones. By the Cauchy-Schwarz inequality, $\left(\mathbf{1}^{T} x\right)^{2} \leq N\|x\|_{2}^{2}$, we find

$$
x^{T} B x \geq\left(\frac{(1-\rho)}{N}+\rho\right)\left(\mathbf{1}^{T} x\right)^{2},
$$

with equality obtained by the choice $x=1$. A necessary and sufficient condition for positive definiteness is therefore

$$
\frac{(1-\rho)}{N}+\rho>0
$$

which leads to the first condition in (15).

Next, we return to the matrix $C$ in which an extra row and column of $m$ 's is appended to $B$, corresponding to the Brownian motion driving the stochastic intensity. We write an arbitrary vector in $\mathbb{R}^{N+1}$ as $\tilde{x}=\left(x^{T} x^{\prime}\right)^{T}$, where $x \in \mathbb{R}^{N}$ and $x^{\prime}$ is a scalar. Then

$$
\begin{aligned}
\tilde{x}^{T} C \tilde{x} & =x^{T} B x+2 m x^{\prime} \mathbf{1}^{T} x+x^{\prime 2} \\
& =(1-\rho)\|x\|_{2}^{2}+\left(\rho-m^{2}\right)\left(\mathbf{1}^{T} x\right)^{2}+\left(x^{\prime}+m \mathbf{1}^{T} x\right)^{2}, \\
& \geq\left(\frac{(1-\rho)}{N}+\left(\rho-m^{2}\right)\right)\left(\mathbf{1}^{T} x\right)^{2}+\left(x^{\prime}+m \mathbf{1}^{T} x\right)^{2},
\end{aligned}
$$

again using the Cauchy-Schwarz inequality. It then follows that a necessary and sufficient condition for positive definiteness is

$$
\frac{(1-\rho)}{N}+\left(\rho-m^{2}\right)>0
$$

which leads to the second condition in (15).

\section{B Diversity Function Computation for Symmetric Model}

In this Appendix, we provide details of the computation of the diversity function in the case of the symmetric model described in Section 2.3. When there are $n$ firms with identical statistics (each has growth rate $\mu$, volatility $\sigma$ and pairwise instantaneous correlation between returns $\rho$ ), the $n \times n$ covariance matrix $\Sigma=\Sigma(n)$ is

$$
\Sigma=\sigma^{2}\left(\begin{array}{ccccccc}
1 & \rho & \rho & \cdot & \cdot & \cdot & \rho \\
\rho & 1 & \rho & \cdot & \cdot & \cdot & \rho \\
\rho & \rho & 1 & \rho & & & \cdot \\
\cdot & & & \cdot & & & \cdot \\
\cdot & & & & \cdot & & \cdot \\
\cdot & & & & & \cdot & \cdot \\
\rho & \cdot & \cdot & \cdot & \cdot & \rho & 1
\end{array}\right) .
$$


Its inverse clearly has the same structure:

$$
\Sigma^{-1}=\frac{1}{\sigma^{2}}\left(\begin{array}{ccccccc}
\alpha & \beta & \beta & . & \cdot & \cdot & \beta \\
\beta & \alpha & \beta & . & . & \cdot & \beta \\
\beta & \beta & \alpha & \beta & & & \cdot \\
\cdot & & & \cdot & & & \cdot \\
\cdot & & & & \cdot & & \cdot \\
\cdot & & & & & . & \cdot \\
\beta & . & . & . & \cdot & \beta & \alpha
\end{array}\right),
$$

where $\alpha$ and $\beta$ must satisfy

$$
\begin{aligned}
\alpha+(n-1) \rho \beta & =1 \\
\beta+\rho \alpha+(n-2) \rho \beta & =0 .
\end{aligned}
$$

These give

$$
\begin{aligned}
\alpha & =\frac{1+(n-2) \rho}{(1-\rho)(1+(n-1) \rho)} \\
\beta & =\frac{-\rho}{(1-\rho)(1+(n-1) \rho)} .
\end{aligned}
$$

Then we compute

$$
D(n)=\mu^{2} \mathbf{1}^{T} \Sigma^{-1} \mathbf{1}=\frac{\mu^{2}}{\sigma^{2}} n(\alpha+(n-1) \beta) .
$$

Finally, using (49) and (50) leads to (16-17).

\section{References}

[1] P. Artzner and F. Delbaen. Default risk insurance and incomplete markets. Mathematical Finance, 5:187-195, 1995.

[2] D. Becherer. Rational hedging and valuation of integrated risks under constant absolute risk aversion. Insurance: Mathematics and Economics, 33(1):1-28, 2003.

[3] T. Bielecki and M. Jeanblanc. Indifference pricing of defaultable claims. In R. Carmona, editor, Indifference Pricing. Princeton University Press, 2006.

[4] T. Bielecki, M. Jeanblanc, and M. Rutkowski. Hedging of defaultable claims. In R. Carmona, editor, Paris-Princeton Lectures on Mathematical Finance. Springer, 2004.

[5] H. Buhlmann. Mathematical Methods in Risk theory. Springer-Verlag, 1970.

[6] P. Collin-Dufresne and J. Hugonnier. Event risk, contingent claims and the temporal resolution of uncertainty. Carnegie Mellon University Working Paper, 2001.

[7] J. Cvitanić, W. Schachermayer, and H. Wang. Utility maximization in incomplete markets with random endowment. Finance and Stochastics, 5(2):259-272, 2001.

[8] M.H.A. Davis and V. Lo. Modelling default correlation in bonds portfolios. Quantitative Finance, 2001.

[9] M.H.A. Davis, V. Panas, and T. Zariphopoulou. European option pricing with transaction costs. SIAM J. Control and Optimization, 31:470-93, 1993.

[10] M. Dempster, E. Medova, and S. Yang. Empirical copulas for CDO tranche pricing using relative entropy. International Journal of Theoretical and Applied Finance, 10(4):679-701, 2007. 
[11] D. Duffie and N. Garleanu. Risk and valuation of collateralized debt obligations. Financial Analysts Journal, 57:41-59, 2001.

[12] D. Duffie, L. Saita, and K. Wang. Multi-period corporate failure with stochastic covariates. Working paper, Stanford University, 2007.

[13] A. Elizalde. Credit risk models IV: Understanding and pricing CDOs. www.abelelizalde.com, 2005.

[14] J.-P. Fouque, B.C. Wignall, and X. Zhou. Modeling correlated defaults: First passage model under stochastic volatility. Journal of Computational Finance, 11(3):43-78, 2008. To appear.

[15] J.P. Fouque, R. Sircar, and K. Sølna. Multiname and multiscale default modeling. Technical report, Department of Mathematics, U.C. Irvine, 2007. Submitted.

[16] H. Gerber. An Introduction to Mathematical Risk Theory. Huebner Foundation for Insurance Education, Wharton School, University of Pennsylvania, 1979.

[17] G. Di Graziano and L.C.G. Rogers. A new approach to the modeling and pricing of correlation credit derivatives. Preprint, University of Cambridge, 2005.

[18] J. Gregory and J.-P. Laurent. Basket default swaps, CDOs and factor copulas. Technical report, BNP Paribas and University of Lyon, 2003.

[19] S.D. Hodges and A. Neuberger. Optimal replication of contingent claims under transaction costs. Review of Futures Markets, 8:222-239, 1989.

[20] J. Hull and A. White. Valuation of a CDO and an nth to default CDS without Monte Carlo simulations. Journal of Derivatives, 12(2):8-23, 2004.

[21] R. Jarrow and S. Turnbull. Pricing options on financial securities subject to credit risk. Journal of Finance, 50:53-85, 1995.

[22] I. Karatzas and S. Shreve. Methods of Mathematical Finance. Springer-Verlag, 1998.

[23] D. Kramkov and W. Schachermayer. The asymptotic elasticity of utility functions and optimal investment in incomplete markets. Annals of Applied Probability, 9(3):904-950, 1999.

[24] D. Lando. On Cox processes and credit risky securities. Review of Derivatives Research, 2:99-120, 1998.

[25] V. Linetsky. Pricing equity derivatives subject to bankruptcy. Mathematical Finance, 16(2):255-282, 2006.

[26] D. Madan and H. Unal. Pricing the risks of default. Review of Derivatives Research, 2:121-160, 1998.

[27] A. Mortensen. Semi-analytical valuation of basket credit derivatives in intensity-based models. Journal of Derivatives, 13(4):8-26, 2006.

[28] E. Papageorgiou and R. Sircar. Multiscale intensity models and name grouping for valuation of multiname credit derivatives. Technical report, Princeton University, 2007. Submitted.

[29] P. Schönbucher. Credit Derivatives Pricing Models. Wiley, 2003.

[30] T. Shouda. The indifference price of defaultable bonds with unpredictable recovery and their risk premiums. Preprint, Hitosubashi University, Tokyo, 2005.

[31] R. Sircar and T. Zariphopoulou. Utility valuation of credit derivatives: Single and two-name cases. In M. Fu, R. Jarrow, J.-Y. Yen, and R. Elliott, editors, Advances in Mathematical Finance, ANHA Series, pages 279-301. Birkhauser, 2007.

[32] C. Tiu. On the Merton Problem in Incomplete Markets. PhD thesis, The University of Texas at Austin, 2002.

[33] O. Vasicek. Probability of loss on loan portfolio. Technical report, KMV Corporation, 1987.

[34] T. Zhou. Indifference valuation of mortgage-backed securities. Preprint, The University of Texas at Austin, 2006. 